\title{
Instantaneous power calculation based on intrinsic frequency of single-phase virtual synchronous generator
}

\author{
Yangyang ZHAO $^{1}$, Jianyun CHAI ${ }^{1}$, Shien WANG $^{1}$, Kai SUN ${ }^{1}$ \\ MPCE
}

\begin{abstract}
In order to enhance the stability of single-phase microgrid, virtual synchronous generator (VSG) control method is investigated in this paper. Its electromagnetic model and electromechanical model are established to illustrate the performance of VSG. Considering the 2nd fluctuation of fundamental-frequency in the output power, an instantaneous power calculation strategy is proposed based on the intrinsic frequency of single-phase VSG. Besides, a virtual power calculation method is presented to achieve islanded/grid-connected seamless transition. Stability analysis and comparison simulation results demonstrate the correctness of the presented power calculation method. At last, the effectiveness of the proposed approach is verified by comparison experiments of islanded/gridconnected operations in a 500 VA single-phase inverter.
\end{abstract}

Keywords Virtual synchronous generator, Single phase inverter, Instantaneous power calculation, Seamless transition

CrossCheck date: 16 January 2017.

Received: 12 October 2015/Accepted: 16 January 2017/Published online: 25 March 2017

(C) The Author(s) 2017. This article is an open access publication

Yangyang ZHAO

zyy900318@126.com

Jianyun CHAI

chaijy@tsinghua.edu.cn

Shien WANG

wang-s-e@163.com

Kai SUN

sun-kai@tsinghua.edu.cn

1 The State Key Lab of Power Systems, Department of Electrical Engineering, Tsinghua University, Beijing, China

\section{Introduction}

Stability of the frequency and voltage in a distributed single-phase microgrid has caught the common attention. As more and more building integrated the energy sources such as photovoltaic, small wind generator, electric vehicle and battery storage system are all connected to a singlephase grid, the stable, reliable and economic operation of this single-phase microgrid are greatly influenced $[1,2]$. Thus, a grid-friendly and improved interface to integrate numerous distributed energy sources into the single-phase microgrid is of much importance.

In order to achieve the proportional power sharing, grid frequency and terminal voltage stabilization and fast islanded/grid-connected seamless transition, some innovative control methods are documented. In [3-6], power sharing of paralleled inverters in the steady state is realized by utilize the $P-f$ and $Q-U$ droop controllers. To improve the dynamic synchronism of frequency, virtual inertia and damping factor are emulated based on derivative of the detected grid frequency in [7-9]. Furthermore, considering the intrinsic regulation capacity of the frequency and voltage, and the self-synchronization features of a conventional synchronous generator, elaborated model of three-phase virtual synchronous generator (VSG) is established in $[10,11]$, and such grid interface is widely recognized in three-phase system. It not only enables the functions of the governor and exciter to realize the frequency and voltage regulation in the steady state, but also provides the rotational inertia to the grid and increases the dynamic stability without using the phase-locked loop (PLL). However, VSG control method in the single-phase microgrid still remains to be a challenging issue. Compared with the three-phase system, the major difference in singlephase grid is the twice fluctuation of fundamental- 
frequency in its output power, thus this feature makes the three-phase instantaneous power theory based on Clark transform and Park transform invalid. Therefore, various numerical strategies are proposed to achieve a fast and accurate instantaneous single-phase output power calculation $[12,13]$.

One method to obtain active and reactive power by calculating the mean value and fluctuation is based on the whole period or half period sampling in [14]. Assuming that the voltage phasor $\boldsymbol{u}$ leads the current phasor $\boldsymbol{i}$ with the phase angle $\theta_{u i}$. The lengths of the phasors equal to their peak values, and the instantaneous values equals to their projections $u_{\alpha}$ and $i_{\alpha}$. Inspired by the rotating coordinates, the virtual $\alpha \beta$ frame is utilized to build up the $90^{\circ}$ phase-shifted phasors, $u_{\beta}$ and $i_{\beta}$. It is well understood that the single-phase active power consists of a constant consumed DC-offset power and a twice fundamental-frequency power periodically flow between the load and the source. As the DC power value always equals to the peak value of the periodic fluctuating power in a single phase system, the active and reactive power can be defined as:

$\left\{\begin{array}{l}P=\frac{|\boldsymbol{u} \| \boldsymbol{i}| \cos \left(\theta_{u i}\right)}{2}=\frac{u_{\alpha} i_{\alpha}+u_{\beta} i_{\beta}}{2} \\ Q=\frac{|\boldsymbol{u}||\boldsymbol{i}| \sin \left(\theta_{u i}\right)}{2}=\frac{u_{\beta} i_{\alpha}-u_{\alpha} i_{\beta}}{2}\end{array}\right.$

Hence, multiple methods are presented to create an orthogonal signal generator (OSG) system, such as Hilbert Transform, inverse Park Transform and Second Order Generalized Integrator (SOGI). However, these methods normally require a synchronization unit to realize OSG under certain frequency, and the transient response and dynamic stability of such methods are dependent on the performance of both OSG function and synchronization function. As the frequency of a converter would vary under different conditions in microgrid, the design of these functions is widely recognized a stringent issue.

In this paper, VSG control is embedded with a virtual synchronous rotational shaft which provides the power calculation module with an instant and accurate frequency signal. Thus, it increases the accuracy of the instantaneous power calculation while maintains its stability and speed. Furthermore, a virtual power calculation (VPC) is presented based on the similar method to achieve the islan$\mathrm{ded} /$ grid-connected seamless transition.

The rest of this paper is organized as follows. The VSG control for the single-phase inverter is presented in Sect. 2. The instantaneous power calculation method based on the intrinsic frequency is proposed in Sect. 3, with comparison simulation, stability analysis and seamless transition experimental results are presented in Sect. 4, followed by conclusions made in Sect. 5.

\section{Single-phase virtual synchronous generator}

Figure 1 shows the general configuration of a singlephase inverter based on VSG method.

As indicated in Fig. 1, there are some similarities between a single-phase synchronous generator and the inverter. Theoretically, the input DC source $U_{D C}$ can mimic the prime mover, and the filter inductance $L_{f}$ in the single-phase inverter can be considered as the inductor of the stator $L_{s t}$ of the generator. Additionally, with the electro-magnetic and electro-mechanical model, virtual governor and exciter model built inside the control system, a single-phase inverter can mimic the characteristic of a real synchronous generator.

\subsection{Electro-magnetic and electro-mechanical models}

According to Faraday's Law, the electro-magnetic model is designed to demonstrate the basic relationship between the stator voltage $u_{i n v}$ and the back electro-motive force $(\mathrm{EMF}) e_{0}$. It can be expressed as:

$\left\{\begin{array}{l}u_{i n v}=-R_{s} i_{o}-L_{s} \frac{\mathrm{d} i_{o}}{\mathrm{~d} t}+e_{0} \\ e_{0}=M_{f} i_{f} \omega \sin \delta\end{array}\right.$

where $u_{i n v}$ is the PWM reference of the inverter; $i_{o}$ is the output current; $M_{f}$ and $i_{f}$ are the magnetic inductance and excitation current respectively; $L_{s}$ and $R_{s}$ are the stator impedance which are the combination of the real existed filter inductor and programmable virtual impedance. Thus,

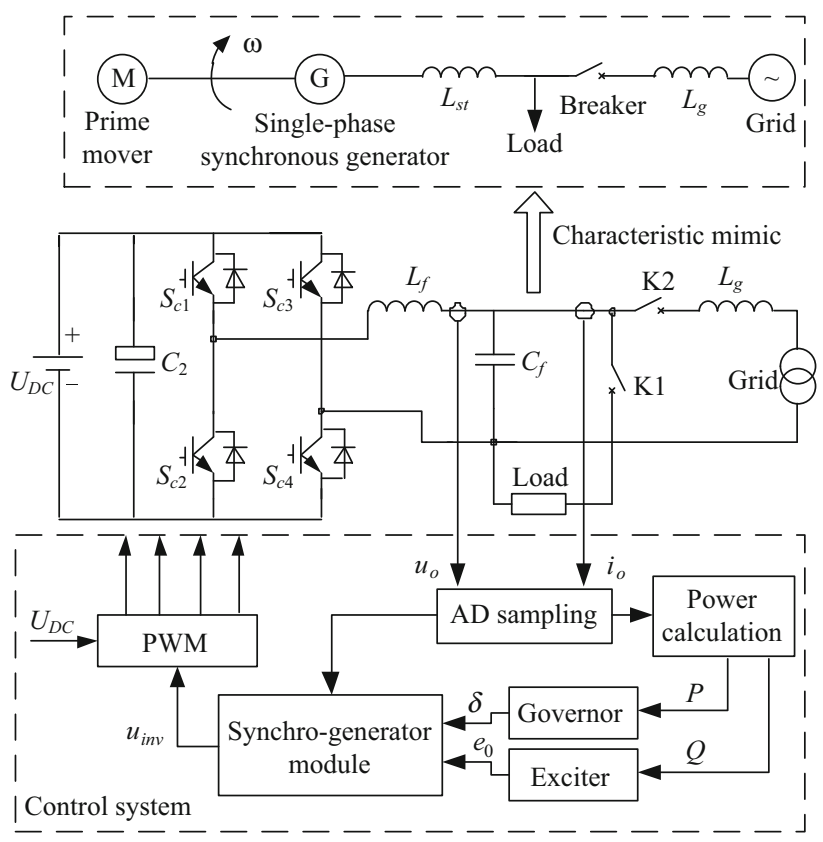

Fig. 1 General configuration of a single-phase VSG 
the output voltage can be coordinated by mimicking the parameter of a real synchronous generator. Especially, $\delta$ is the power angle which is determined by the electro-mechanical model, and therefore it provides the inverter with the capacity to generate the synchronous frequency $\omega$.

According to Newton's Law, the electro-mechanical model is designed to illustrate the motion modes of generator. It can be expressed as:

$J \alpha=T_{m}-T_{e}+D \omega$

where $T_{m}$ and $T_{e}$ are the mechanical torque and electromagnetic torque, respectively; $D$ is the rotor damping factor; $J$ is the virtual inertia; $\alpha$ is the angular acceleration; and $\omega$ is the synchronous frequency which also represents the dynamic rotating speed of the back EMF. The virtual synchronous shaft is built up through the equation above to represent the electro-mechanical energy conversion process of a real generator [11]. Therefore, not only the voltagecurrent relation of inverter meets the feature of a synchronous generator, but also the frequency dynamic response conforms to the rotor motion rule inside the generator. The synchronous generator module provides inverter with the flexibility to mimic various rotor inertia constants, stator impedance to achieve the similar interface of single-phase generators of same power level.

\subsection{Virtual governor and virtual exciter}

On the basis of the proposed generator module above, virtual governor and virtual exciter are employed to realize the ability of the frequency and voltage regulation in the real synchronous generator system. The active power-frequency static feature is established on the $P-f$ droop controller, and the reactive power-voltage static feature is established on the $Q-U$ droop controller. Both controllers can be expressed as:

$$
\left\{\begin{array}{l}
D_{P}=\frac{\Delta f}{\Delta P}=\frac{\left(f-f_{n}\right) / f_{n}}{\left(P_{n}-P_{r e f}\right) / P_{n}} \\
D_{Q}=\frac{\Delta U}{\Delta Q}=\frac{\left(U_{o}-U_{n}\right) / U_{n}}{\left(Q_{n}-Q_{r e f}\right) / Q_{n}}
\end{array}\right.
$$

where $D_{P}$ and $D_{Q}$ are the $P-f$ droop factor and the $Q-U$ droop factor respectively; $P_{n}, f_{n}, Q_{n}, U_{n}$ are the nominal values of active power, frequency, reactive power and voltage, respectively; According to the equations, $P_{\text {ref }}$ represents the input mechanical power reference under present frequency $f$ to enable active load sharing between paralleled inverters, while $Q_{r e f}$ represents the reactive power reference under present voltage amplitude $U_{o}$, as well as realizes the proportional reactive power sharing. It is highlighted that the present frequency can be obtained from the proposed virtual rotor shaft above without using any dedicated PLL, and the virtual inertia increases the dynamic stability of the synchronous frequency in the single-phase microgrid.

\subsection{VPC-based seamless transition}

Though the single-phase VSG is a voltage-controlled source which owns the advantages of the self-regulated frequency and voltage, a fast and adequately accurate synchronization process remains to be a stringent issue. It is well understood that PLL may lead to heavy calculation burden and time-consuming when there is a phase jump event or sudden frequency change occurs. Supposing there is virtual impedance between the grid and the inverter, virtual current can be calculated as:

$i_{v}=\frac{u_{o}-u_{g}}{L_{v} s+R_{v}}=\frac{|\Delta u| \angle \delta_{g}}{L_{v} s+R_{v}}$

where $u_{o}$ and $u_{g}$ are the instant value of inverter output voltage and grid voltage; $L_{v}$ and $R_{v}$ are virtual synchronizing impedance; $i_{v}$ is the virtual current which represents the virtual power flowing between the inverter and the grid, while $\delta_{g}$ represents the virtual power angle. Then, $u_{o}$ and $i_{v}$ are applied to calculate the virtual power during the presynchronization process. And the completion flag of such process can be recognized when the VPC result is near zero. The control module of such VPC-based seamless transition is depicted in Fig. 2.

Note that VPC method displaces the function of a PLL unit, thus provides the possibility of the seamless transition in a single-phase VSG system. The operation methods of the islanded mode, grid pre-synchronization and transition processes are introduced in the followings.

When all three switches in Fig. 2 are set to Position 1, the VSG operates under the islanded mode, in which the virtual governor undertakes the function to generate a

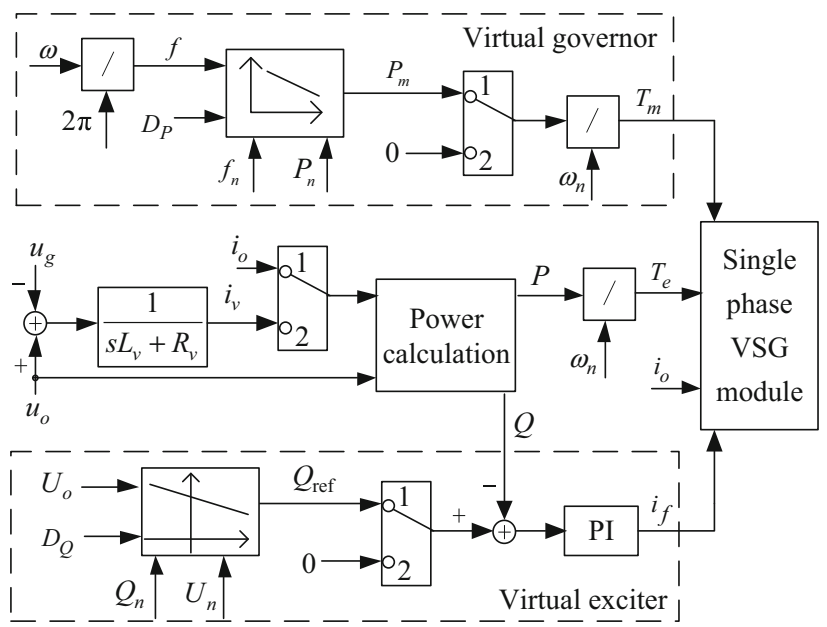

Fig. 2 VPC-based seamless transition control module for VSG 
mechanical torque $T_{m}$ with due $P-f$ droop factor, while the virtual exciter can generate the excitation current $i_{f}$ with due $Q-U$ droop factor. Thus, both the local frequency and voltage can be coordinated in islanded mode.

When the inverter receives the command to connect to a grid, all the switches in Fig. 2 are thrown to Position 2. In the virtual governor block, the mechanical power reference $P_{m}$ is set to zero, and the virtual current $i_{v}$ is implemented to calculate the virtual power. If virtual power is positive while inverter voltage phasor leads the grid voltage phasor, intrinsic frequency $f$ of VSG will decrease, and vice versa. By calculating the virtual power flow between the inverter and grid, pre-synchronization can be realized. Then, phase breaker can be turned on as soon as the calculated virtual power is close to zero with grid-connected current under well-controlled. Considering the virtual inertia $J$ which is set in the VSG module may lag the pre-synchronization time, an alternative switch can be embedded in the VSG module to deprive inertia constant whenever a fast grid synchronization is in need. Furthermore, small value of $L_{v}$ and $R_{v}$ can also accelerate the synchronization process, but may also lead to oscillations in the frequency estimation.

After being connected to the grid, all the switches are thrown back to Position 1 with the actual output power can be regulated again in the VSG control model. In case of a drastically sudden power change occurs, an uploading process is alternative under certain circumstances.

Whether being connected to a strong grid or a weak grid, the single-phase VSG can operate in the similar way as it does in the islanded mode. After the uploading process, the output power of the inverter is fixed according the initial nominal power $P_{n}$ and $Q_{n}$ set in the $P-f$ and $Q-U$ droop controllers. However, when the frequency or voltage of the grid varies, the power references can also be affected according to (4).

It is easy to illustrate when VSG connects to a strong grid with fixed frequency and voltage. As the active power flow is determined by the power angle, and the power angle can be controlled by the virtual mechanical torque, the variation of nominal active power $P_{n}$ can move the $P-f$ droop curve vertically which enable the output active power $P$ tracks its command. Similarly, as the reactive power flow is determined by the excitation current, and the current can be controlled by the voltage error, the variation of the nominal reactive power $Q_{n}$ can move the $Q-U$ droop curve vertically which enable the output reactive power $Q$ tracks its command. Additionally, when VSG connects to a weak grid or even other VSGs with floating frequency and voltage, the control strategy of injection power remains the same as is mentioned above. Thanks to the VSG belongs to the synchronous power source which has intrinsic regulation ability of both frequency and voltage, the frequency and voltage of the point of common coupling
(PCC) are determined by all the paralleled power source, while the $P-f$ and $Q-U$ droop controllers enable the proportional power sharing.

However, considering the frequency is variable by adopting the VSG control, the single-phase power calculation is more challenging and stringent. What's more, both real power calculation and virtual power calculation requires the dynamic stability, accuracy and appropriate calculation speed to ensure a high performance of the VSG. Hence, taking the advantage of VSG control method, an innovative instantaneous single-phase power calculation is of great significance.

\section{Single-phase power calculation based on intrinsic frequency}

The main task for calculating instantaneous power in single-phase system is to extract average and fluctuating components from the cycles of sine waves. On the basis of SOGI which is a good alternative for creating orthogonal system in [7], the proposed method utilizes the intrinsic frequency signal from VSG module to achieve a more accurate and stable power calculation.

The sampled output voltage and current $u_{o}, i_{o}$ can be transferred into two groups of quadrature signals $u_{\alpha}, u_{\beta}$ and $i_{\alpha}, i_{\beta}$ through SOGI system. Introduced with the intrinsic frequency from the virtual shaft of the single-phase VSG module, the closed-loop transfer functions of SOGI are defined as:

$$
\left\{\begin{array}{l}
H_{\alpha}(s)=\frac{u_{\alpha}}{u}(s)=\frac{i_{\alpha}}{i}(s)=\frac{k \omega s}{s^{2}+k \omega s+\omega^{2}} \\
H_{\beta}(s)=\frac{u_{\beta}}{u}(s)=\frac{i_{\beta}}{i}(s)=\frac{k \omega^{2}}{s^{2}+k \omega s+\omega^{2}}
\end{array}\right.
$$

From the perspective of frequency-domain, the two output signals with a phase shift of $90^{\circ}$ are generated, while the component on $\alpha$ axis has the same phase and magnitude as the fundamental of input signal. And the gain factor $k$ affects the bandwidth of the closed-loop system which will be analyzed in detail in the following. Commonly, the resonance frequency $\omega$ is set on the system nominal value $\omega_{n}$. From the perspective of time-domain, the proposed instantaneous power calculation actually utilize the information of $1 / 4$ fundamental cycles of voltage and current, and can give out a refreshed result every $1 \mathrm{AD}$ interruption cycle. However, as the proposed SOGI structure is frequency dependent, problems can occur when system frequency fluctuating under different conditions. Thanks to the VSG module can generate the continuously and slowly-varied intrinsic frequency, it can be employed easily without the fear of frequency skipping or phase jumping event. Here, the resonance frequency $\omega$ 
can be replaced with the intrinsic frequency of VSG, and this embedded value assures the accuracy of center frequency without need of any PLL, thus improves the performance of instantaneous power calculation with SOGI method.

\subsection{Actual power calculation}

To utilize the grid-friendly interface, the VSG control strategy in single-phase system is proposed above. Therefore, the frequency of the inverter is flexible under different operation modes, while the center frequency deviation in OSG system can cause the inaccurate power calculation in both dynamic and steady state. The Bode diagram of SOGI system is shown in Fig. 3a.

The magnitude-frequency curves indicate the band-pass feature of $H_{\alpha}(s)$ and low-pass feature of $H_{\beta}(s)$. The phasefrequency curves indicate the $0^{\circ}$ shift feature of $H_{\alpha}(s)$ and $90^{\circ}$ shift feature of $H_{\beta}(s)$ in the center frequency. It is easily understood that only the resonance frequency equals to the frequency of detected sine waves, the instantaneous

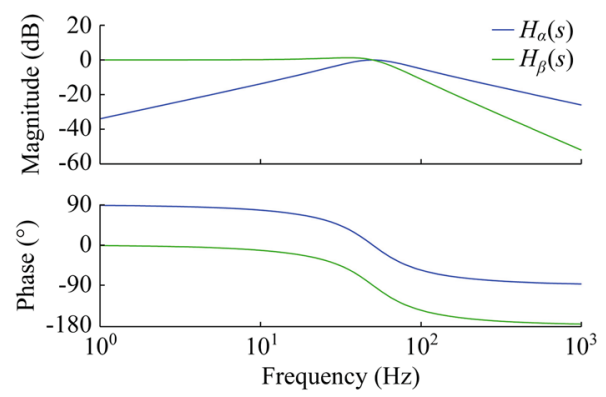

(a) Bode diagram of closed-loop SOGI system

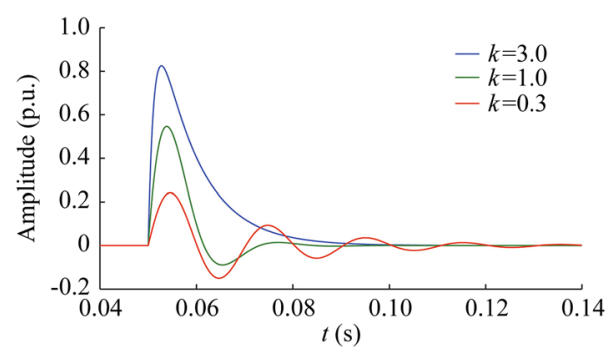

(b) Step response of SOGI system under different gain factors

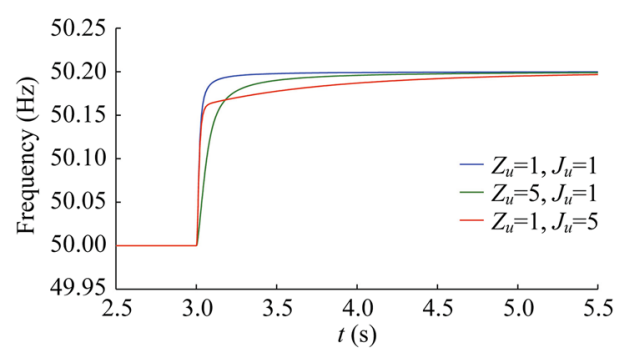

(c) Dynamic response of virtual power calculation

Fig. 3 Dynamic responses of the proposed power calculation method power calculation under SOGI method can generate a correct result. The larger the frequency deviation is, the more phase-shift and amplitude decay occurs. Therefore, the program embedded feature and continuity feature of the intrinsic frequency can provide the advantage to calculate more accurate instantaneous power while maintain the dynamic stability of the original SOGI method. While in the conventional method, the constant resonance frequency would lead to an imprecise power result.

It is obvious that with the decrease of $k$, bandwidths are narrowed both in the magnitude and phase diagrams. Hence, in order to avoid too much magnitude decay and phase shift when the frequency deviates from the center frequency, conventional SOGI system requires a slightly higher $k$ which in turn leads to a larger overshot in step response result. Step response figure of $H_{\alpha}(s)$ at different gain factors is shown in Fig. 3b. In general, lots of efforts need to be taken to balance the frequency bandwidth and overshot in the conventional SOGI system. While the proposed instantaneous power calculation based on an intrinsic and comparatively slow changing frequency can provide the power computation process with desired small overshot and considerably narrow bandwidth around the varied intrinsic frequency. In this paper, $k$ is equal to 1.0 for the simulation and the experiment.

\subsection{Virtual power calculation}

As is mentioned above, stability of the power calculation module plays an important role in seamless transition based on the virtual power strategy. Similar to the actual power calculation method, the performance of virtual power calculation can also be elevated based on the intrinsic frequency. Conventionally, PLL unit is always considered as time-consuming and unstable under sudden frequency change or phase jump events. Even though advanced PLL designs have solved some of the issues, the present paralleled power system actually contains multiple frequencies as each VSG or frequency-voltage regulated source injects its own intrinsic frequency into grid, therefore, makes it more troublesome for PLL unit to generate a stable grid frequency during the pre-synchronization period.

Apparently, the intrinsic frequency serves as the stable and accurate information for center frequency in SOGI system, which allows virtual power calculation to be near zero even under varied grid frequency. Though implementing the same SOGI system, the virtual synchronizing impedance and the virtual inertia also play significant roles during the pre-synchronization period. Figure $3 \mathrm{c}$ shows the dynamic response of the intrinsic frequency based SOGI system under different virtual synchronizing impedance and virtual inertia where $k$ of SOGI 
system is equal to 1.0. In the frequency response waveforms in Fig. 3c, $Z_{u}$ is per unit value of virtual synchronizing impedance and $J_{u}$ is per unit value of virtual inertia. When a sudden change of the grid frequency from 50 to $50.2 \mathrm{~Hz}$ occurs in $3 \mathrm{~s}$, varied frequency can be tracked smoothly according to the proposed intrinsic frequencybased VPC. Additionally, the regulation time can be extended along with the increase of $Z_{u}$ and $J_{u}$. It is obvious that $Z_{u}$ functions as the ratio factor for calculated errors of the virtual power from zero, increasing the virtual synchronizing impedance would prolong the synchronization time proportionally, while $J_{u}$ determines the changing speed of the intrinsic frequency. Thus, the smaller virtual inertia is, the shorter the synchronization period would take. Firstly, small value of $L_{v}$ and $R_{v}$ can accelerate the synchronization process, but may also lead to oscillations in frequency estimation. Secondly, the ratio of $R_{v} / L_{v}$ defines the cut-off frequency of output voltage, which determines the capacity of filtering out the harmonics in grid voltage. Thus, $L_{v}$ and $R_{v}$ can be set slightly smaller than stator impedance $L_{s}$ and $R_{s}$.

As SOGI system based on the intrinsic frequency method is implemented in both actual and virtual power calculations, it provides the possibility for the single-phase VSG to achieve seamless transition.

\section{Simulation and experiment results}

\subsection{Simulation results}

The proposed instantaneous power calculation based on intrinsic frequency of single-phase VSG is verified with simulations carried out in MATLAB/Simulink. Comparison simulations are also designed with conventional power calculation based on the nominal frequency. Parameters of VSG and control system are all given in Table 1.

The PWM carrier wave frequency and sampling frequency are chosen to be $10 \mathrm{kHz}$. Virtual inertia of the proposed VSG is chosen to be $0.001 \mathrm{~kg} / \mathrm{m}^{2}$ which stand for

Table 1 Parameters used in simulation and experiment

\begin{tabular}{llll}
\hline Parameters & Values & Parameters & Values \\
\hline$L_{s}$ & $0.45 \mathrm{mH}$ & $P_{n}$ & $250 \mathrm{~W}$ \\
$R_{s}$ & $0.13 \Omega$ & $Q_{n}$ & $250 \mathrm{var}$ \\
$C_{f}$ & $22 \mu \mathrm{F}$ & $f_{n}$ & $50 \mathrm{~Hz}$ \\
$L_{v}$ & $0.2 \mathrm{mH}$ & $U_{n}$ & $230 \mathrm{~V}$ \\
$R_{v}$ & $0.05 \Omega$ & $U_{D C}$ & $350 \mathrm{~V}$ \\
$L_{g}$ & $0.05 \mathrm{mH}$ & $D_{P}$ & 0.01 \\
$J_{v}$ & $0.001 \mathrm{~kg} / \mathrm{m}^{2}$ & $D_{Q}$ & 0.01 \\
\hline
\end{tabular}

the inertia constant $H_{v}=0.2 \mathrm{~s}$ in the per unit system according to:

$H_{v}=\frac{1}{2} J_{v} \omega_{n}^{2} / P_{n}$

Simulation circuit is exactly the same as shown in Fig. 1 where the full-bridge single-phase inverter is connected to a local load via the local breaker $\mathrm{K} 1$, as well as connected to the grid via the grid breaker K2. $P-f$ droop factor $D_{P}=0.01$ is chosen so that $1 \%$ nominal frequency variation correspondent to $100 \%$ nominal active power, and $Q-U$ droop factor $D_{Q}=0.01$ is chosen so that $1 \%$ nominal voltage variation correspondent to $100 \%$ nominal reactive power. With the turn on/off combinations of the breakers $\mathrm{K} 1$ and $\mathrm{K} 2$, simulations under islanded mode, grid-connected mode and transition operation modes are elaborated in the following.

In islanded mode, $\mathrm{K} 1$ is enabled and $\mathrm{K} 2$ remains turnoff according to main circuit shown in Fig. 1, while control switches in Fig. 2 are in Position 1. VSG starts with noload mode while $261 \Omega$ resistive load is connected at $0.4 \mathrm{~s}$. The output power and frequency waveforms under intrinsic frequency power calculation and conventional power calculation are shown in Fig. 4a, b, respectively. It can be seen that frequency varies from $50.5 \mathrm{~Hz}$ to $50.05 \mathrm{~Hz}$ along with active power changes from 0 to $225 \mathrm{~W}$ in Fig. $4 \mathrm{a}$. While in Fig. 4b, frequency drops to $50.08 \mathrm{~Hz}$ which is higher than the regulated frequency according to the droop curves. As resonance frequency of power calculation in conventional method deviates from the actual frequency, power calculation results are smaller than the actual power with the same load and terminal voltage. Thus, the

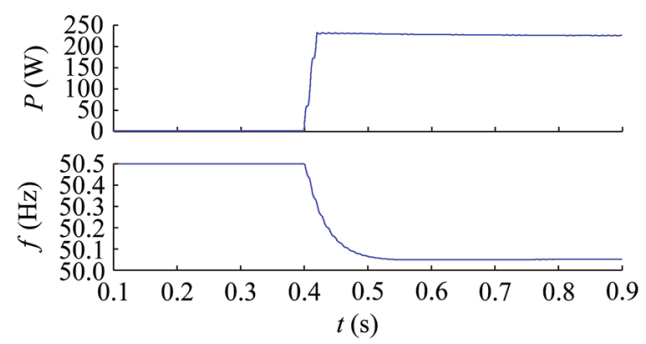

(a) $P$ - $f$ curves under intrinsic frequency power calculation

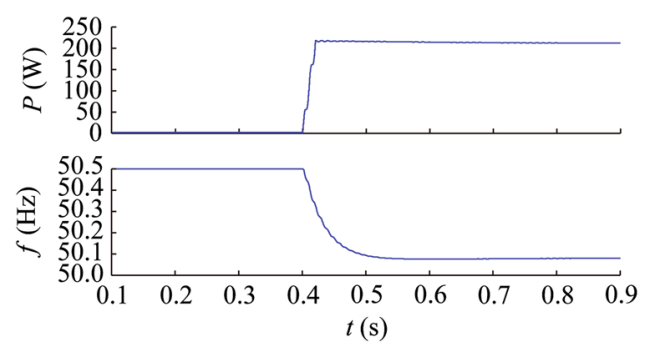

(b) $P$ - $f$ curves under conventional power calculation

Fig. 4 Comparison of simulation results under islanded mode 
regulated frequency according to the droop curves becomes larger than its expected value.

In grid-connected mode, $\mathrm{K} 2$ is closed-on and $\mathrm{K} 1$ remains turn-off according to the main circuit shown in Fig. 1, while control switches in Fig. 2 are all in Position 1. Simulation of grid-connected mode is conducted in the following steps. Firstly, VSG is connected to a grid $(50 \mathrm{~Hz}$, $230 \mathrm{~V}$ ) while injecting an active power of $250 \mathrm{~W}$. Secondly, sudden change of the grid frequency increases from 50.0 to $50.1 \mathrm{~Hz}$ at $0.62 \mathrm{~s}$.

Figure 5 reveals the dynamic response of the grid-connected VSG under grid frequency sudden change event. As a virtual synchronous shaft is introduced, though grid frequency increases abruptly, power angle between VSG and the grid opens up gradually according to the virtual inertia, thus no power impact occurs and VSG could follow the grid within $0.2 \mathrm{~s}$. According to the $P-f$ droop control, the injected power would drop to $200 \mathrm{~W}$ in the steady state which is shown in Fig. 5a. While in Fig. 5b, injected power drops to $223 \mathrm{~W}$ which is larger than the regulated one. As the power calculation in Fig. 5a applies the intrinsic frequency of VSG which makes the resonance frequency follows the actual frequency, there is no attenuation of the calculated power value. As the grid capacity is much stronger than inverter, and the frequency is set to $50.1 \mathrm{~Hz}$, conventional power calculation under nominal frequency is no longer accurate. Smaller calculation results in the digital system leads to a larger power injection in the analog system.

Seamless transition simulation is conducted as follows. Firstly, VSG is under no-load operation with K1 and K2 are both turn-off, and control switches are all in Position 1, grid frequency and voltage are equal to their nominal value, which are $50 \mathrm{~Hz}$ and $230 \mathrm{~V}$. At $0.25 \mathrm{~s}$, VSG

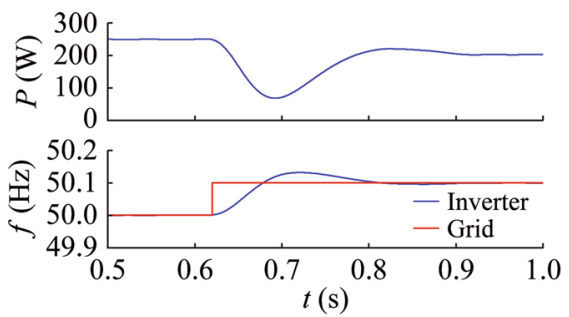

(a) $P-f$ curves under intrinsic frequency power calculation

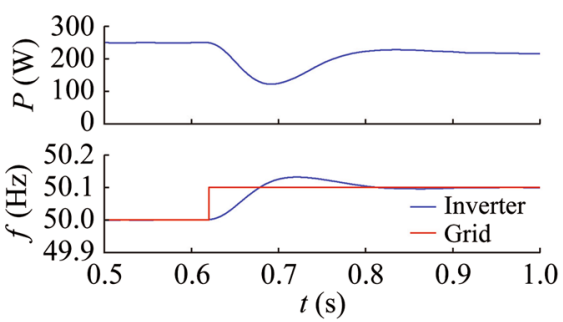

(b) $P$ - $f$ curves under conventional power calculation

Fig. 5 Comparison of simulation results under grid-connected mode receives pre-synchronization command with control switches are thrown to Position 2. When the voltage error between both sides of $\mathrm{K} 2$ is considerably small, $\mathrm{K} 2$ is closed to connect VSG to the grid. Then, the switches are thrown back to Position 1 to output $250 \mathrm{~W}$ and 250 var.

Comparison simulation results are shown in Fig. 6. The grid voltage $u_{g}$, the output voltage $u_{o}$ and current $i_{o}$ are depicted to illustrate the differences between the proposed power calculation methods and conventional methods. It can be observed from Fig. 6a that there is no inrush current $i_{o}$ occurs between the grid and the inverter during islanded to grid-connected mode transition which verifies the proposed virtual power calculation can achieve a seamless transition without PLL. However, Fig. 6b reveals an inrush current up to 4.8 A during the transition period.

And the major difference comes from the resonance frequency in virtual power calculation module. As frequency in no-load mode is $50.5 \mathrm{~Hz}$, in order to synchronize with grid frequency $50 \mathrm{~Hz}$, inverter would gradually decrease its output frequency. As the conventional calculation using the nominal frequency as its resonance frequency, it leads to attenuation in calculated results, which may cause grid breaker close in advance when virtual power is not smaller enough, then higher inrush current is generated.

\subsection{Experiment results}

The proposed power calculation method is also tested with a 500 VA single-phase VSG. The parameters used in the experiments which are similar to the simulations are shown in Table 1. The switching frequency and sampling frequency are chosen as $10 \mathrm{kHz}$.

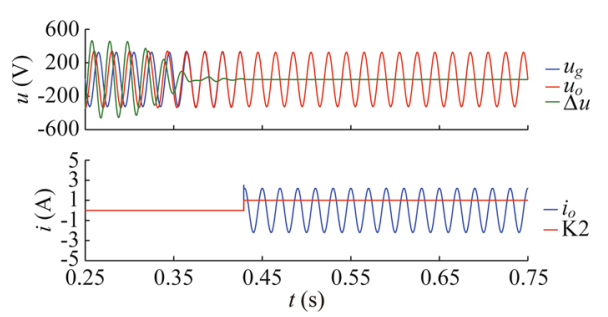

(a) Voltage and current under intrinsic frequency power calculation

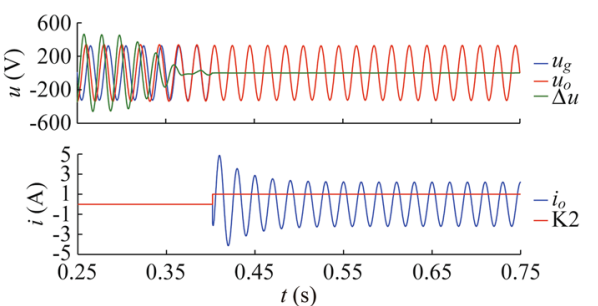

(b) Voltage and current under conventional power calculation

Fig. 6 Comparison of simulation results under seamless transition 
Firstly, to verify the advantages of the proposed intrinsic frequency-based actual power calculation, comparison experiment under the islanded mode is conducted as follows: firstly, a $225 \mathrm{~W}$ load impacts at $2.5 \mathrm{~s}$, then a $225 \mathrm{~W}$ load drops at $7.5 \mathrm{~s}$. Waveforms of the output active power and frequency are extracted from the recorded sinusoid voltage and current data, and then depicted in Fig. 7. It is obvious that $50.05 \mathrm{~Hz}$ should correspond to $225 \mathrm{~W}$ according to the $P-f$ droop curves as shown in Fig. 7a, while in Fig. 7b, the frequency under conventional method only drops to $50.09 \mathrm{~Hz}$. Thanks to the intrinsic frequency of VSG, the generated orthogonal phasor in the singlephase power calculation module would not have damped amplitude compared with the conventional method. However, as the resonant frequency of conventional power calculation never matches the real output frequency during the frequency decrease period in Fig. $7 \mathrm{~b}$, the calculated power is always smaller than the real power. Thus, the smaller calculated power in control system would correspond to a larger frequency than its expected value. Then, the $P-f$ relationship would not coincide with the droop requirements when applies the conventional power calculation method.

Moreover, to illustrate the merits of the VPC-based seamless transition, comparison experiment of the islanded/grid-connected transition is conducted. The actions of all the breakers and switches are in accordance with those in the correspondent simulation.

The islanded/grid-connected transition results are depicted in Fig. 8. As grid frequency stays on $50 \mathrm{~Hz}$ and its

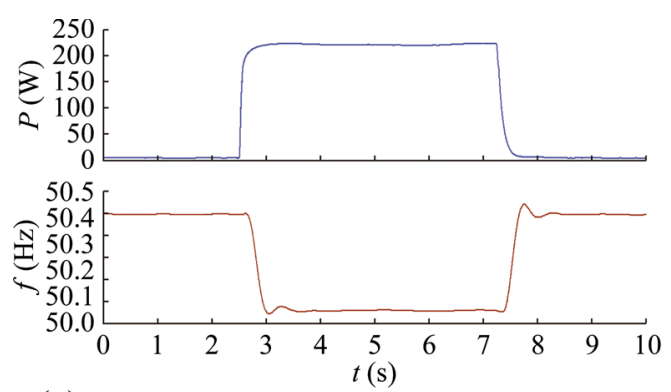

(a) $P$ - $f$ curves under intrinsic frequency power calculation

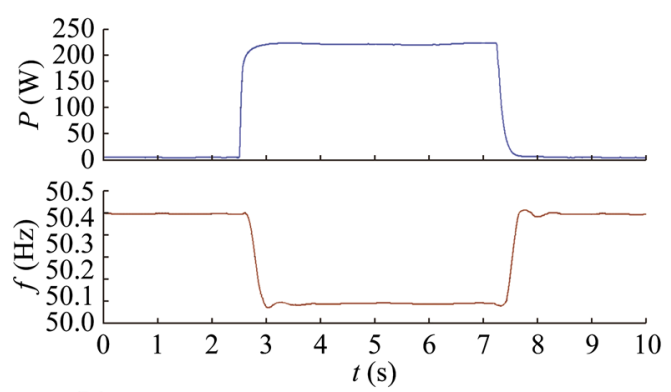

(b) $P$-f curves under conventional power calculation

Fig. 7 Comparison of experimental results under islanded mode

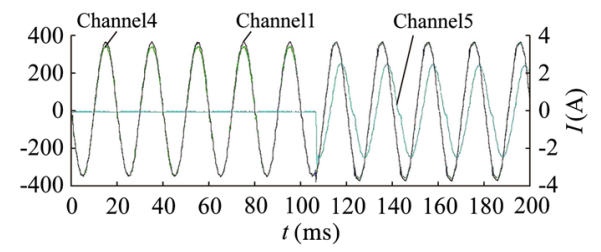

(a) Voltage and current under intrinsic frequency power calculation

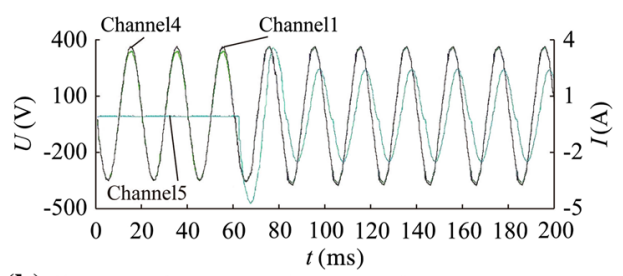

(b) Voltage and current under conventional power calculation

Fig. 8 Comparison of experimental results under seamless transition

voltage is $230 \mathrm{~V}$, the active power and reactive power injecting is constant as $250 \mathrm{~W}$ and 250 var according to the droop curves.

It is clear that the peak of the inrush current during the transition period is regulated within $3 \mathrm{~A}$ in Fig. 8a, which adopts the proposed virtual power calculation embedded with the intrinsic frequency. Moreover, the transition time is constrained inside $10 \mathrm{~ms}$ with no overshot or oscillation occurs. While also applying the virtual power calculation method, the inrush current peak is up to $4.8 \mathrm{~A}$ in Fig. 8b, which only adopts the conventional power calculation with a constant resonant frequency. Thus, the damped amplitude in the calculated virtual power value causes the grid breaker being closed ahead of time, and lead to a much larger inrush current.

\section{Conclusion}

In order to achieve the proportional power sharing, frequency and voltage stabilization, as well as a fast gridtied/islanded transition in the single-phase microgrid, an accurate and fast power calculation method is significant. On the basis of the intrinsic frequency generated from electro-mechanical model of VSG, an instantaneous singlephase power calculation strategy is developed. As the frequency value can be updated every control cycle in microgrid, this method can provide converter with faster and more precise power calculation results. Besides, a virtual power calculation method is also presented to achieve the islanded/grid-connected seamless transition without PLL, and its pre-synchronization time can be decreased by means of the adaptive virtual inertia. Then, the dynamic response performance of the proposed method is illustrated by the stability analysis. Moreover, comparison simulations also demonstrate its calculation speed and 
accuracy. The advantages of the proposed methods over conventional power calculation based on nominal frequency are verified through islanded operation and islanded/grid-connected transition experiments. It is explicit that the intrinsic frequency of VSG can provide the power calculation with a more accurate resonant frequency than conventional method. And with more accurate calculated power value, inverter can acquire better performance to meet droop requirements in islanded mode, as well as inject accurate power into the grid in the grid-connected mode. What's more, the inrush current can also be better regulated during the islanded/grid-connected transition with the more precise virtual power calculation results.

However, as the intrinsic frequency of VSG model is regulated by the virtual inertia, its dynamic processes which have shorter time constants such as electrical magnetic process are not taken into consideration in this model. Therefore, the power regulation in the electrical constants dynamic process and higher harmonics is the further focus of the research.

Acknowledgements This work is supported by the National Basic Research Program of China (973 Program) (No. 2013CB02708201).

Open Access This article is distributed under the terms of the Creative Commons Attribution 4.0 International License (http:// creativecommons.org/licenses/by/4.0/), which permits unrestricted use, distribution, and reproduction in any medium, provided you give appropriate credit to the original author(s) and the source, provide a link to the Creative Commons license, and indicate if changes were made.

\section{References}

[1] Zhong QC, Hornik T (2012) Control of power inverters in renewable energy and smart grid integration. Wiley, New York

[2] Blaabjerg F, Teodorescu R, Liserre M et al (2006) Overview of control and grid synchronization for distributed power generation systems. IEEE Trans Ind Electron 53(5):1398-1409

[3] Rocabert J, Luna A, Blaabjerg F et al (2012) Control of power converters in AC microgrids. IEEE Trans Power Electron 27(11):4734-4749

[4] Guan Y, Wu W, Guo X et al (2010) An improved droop controller for grid-connected voltage source inverter in microgrid. In: Proceedings of the 2nd IEEE international symposium on power electronics for distributed generation systems, Hefei, China, 16-18 Jun 2010, 6 pp

[5] Vandoorn TL, De Kooning JDM, Meersman B et al (2012) Automatic power-sharing modification of/droop controllers in low-voltage resistive microgrids. IEEE Trans Power Deliv 27(4):2318-2325

[6] Guerrero JM, Matas J, De Vicuna LG et al (2006) Wirelesscontrol strategy for parallel operation of distributed-generation inverters. IEEE Trans Ind Electron 53(5):1461-1470
[7] Van Wesenbeeck MPN, De Haan SWH, Varela P et al (2009) Grid tied converter with virtual kinetic storage. In: Proceedings of the 2009 IEEE Bucharest powertech conference, Bucharest, Romania, 28 June-2 July 2009, 7 pp

[8] Arani MFM, El-Saadany EF (2013) Implementing virtual inertia in DFIG-based wind power generation. IEEE Trans Power Syst 24(2):1373-1384

[9] Alipoor J, Miura Y, Ise T (2015) Power system stabilization using virtual synchronous generator with alternating moment of inertia. IEEE J Emerg Sel Top Power Electron 3(2):451-458

[10] Zhong QC, Nguyen PL, Ma Z et al (2014) Self-synchronized synchronverters: inverters without a dedicated synchronization unit. IEEE Trans Power Electron 29(2):617-630

[11] Zhong QC, Weiss G (2011) Synchronverters: inverters that mimic synchronous generators. IEEE Trans Ind Electron 58(4):1259-1267

[12] Xue Y, Chang L, Kjaer SB et al (2004) Topologies of singlephase inverters for small distributed power generators: an overview. IEEE Trans Power Electron 19(5):1305-1314

[13] Zeng Z, Zhao R, Yang H et al (2013) Single-phase virtual synchronous generator for distributed energy sources. In: Proceedings of the 2013 international conference on electrical machines and systems (ICEMS), Busan, South Korea, 26-29 Oct 2013, 6 pp

[14] Dasgupta S, Sahoo S, Panda SK (2011) Single-phase inverter control techniques for interfacing renewable energy sources with microgrid-part I: parallel-connected inverter topology with active and reactive power flow control along with grid current shaping. IEEE Trans Power Electron 26(3):717-731

Yangyang ZHAO received his B.S. degree in Electrical Engineering from Nanjing University of Aeronautics and Astronautics, China, in 2008. Currently he is a Ph.D. candidate in Electrical Engineering in Tsinghua University, China. His major fields of study are control and stability analysis of grid-integrated wind turbines, virtual synchronous control of power converters and microgrid modeling and simulation.

Jianyun CHAI received a B.S. degree and a Ph.D. in Electrical Engineering from Tsinghua University, China, in 1984 and 1989, respectively. He is now a Professor in the Department of Electrical Engineering, Tsinghua University. His research interests include special electrical machine system, power electronic transformer energy manage system and control technologies in wind energy generation.

Shien WANG received his B.S. in Electrical Engineering from Tsinghua University, China, in 2015. He is currently pursuing his $\mathrm{Ph} . \mathrm{D}$. degree in Electrical Engineering there. His research interests include high power DC/DC converter, virtual synchronous generator interface and power electronic transformer.

Kai SUN received a B.S. degree and a Ph.D. in Electrical Engineering from Tsinghua University, China, in 2000 and 2006, respectively. He is now an Associate Professor in the Department of Electrical Engineering, Tsinghua University. His research interests include control and grid-integration of renewable energy generations, hybrid $\mathrm{AC} / \mathrm{DC}$ microgrid and multi-level power converters. 